\title{
Association of VEGF Gene Polymorphisms with Susceptibility to Diabetic Retinopathy: A Systematic Review and Meta-Analysis
}

Authors

Qianhui Yang, Yan Zhang, Xiaomin Zhang, Xiaorong Li*, Juping Liu*

\begin{abstract}
Affiliation
Tianjin Key Laboratory of Retinal Functions and Diseases, Tianjin Medical University Eye Hospital, Tianjin, China
\end{abstract}

Key words

VEGF, gene polymorphism, diabetic retinopathy, metaanalysis, type 2 diabetes

$\begin{array}{lr}\text { received } & 13.11 .2018 \\ \text { accepted } & 11.03 .2020\end{array}$

Bibliography

DOI https://doi.org/10.1055/a-1143-6024

Horm Metab Res 2020; 52: 264-279

(c) Georg Thieme Verlag KG Stuttgart · New York ISSN 0018-5043

\section{Correspondence}

Xiaorong Li MD, PhD

Tianjin Medical University Eye Hospital, Tianjin Medical University Eye Institute, College of Optometry and

Ophthalmology, Tianjin Medical University

300384 Tianjin

China

lixiaorong@tmu.edu.cn

\section{Juping Liu MD}

Tianjin Medical University Eye Hospital, Tianjin Medical University Eye Institute, College of Optometry and Ophthalmology, Tianjin Medical University

300384 Tianjin

China

Tel.: + 862286428808 , Fax: + 862286428777

tydljp@126.com

\section{ABSTRACT}

The associations between vascular endothelial growth factor (VEGF) gene polymorphisms and risk of type 2 diabetic retinopathy (DR) - proliferative diabetic retinopathy (PDR), and nonproliferative diabetic retinopathy (NPDR) - remain unclear. A systematic search and meta-analysis using odds ratio (OR) with $95 \%$ confidence interval $(\mathrm{Cl})$ was performed to evaluate the association. Our study concluded 26 studies containing 10 single nucleotide polymorphisms (SNPs). In Asian populations, rs3025039 polymorphism was associated with DR risk, while in overall populations and Caucasians, the DR risk was increased by association with rs2010963. There was a significant association between rs 25648 and rs833061 and DR risk in Caucasians. DR risks were found to be significantly associated between rs3025021, rs13207351, and rs2146323 in either overall populations, Caucasians or Asians. Besides, in overall and Asian populations, rs699947 and rs3025039 were associated with PDR risk. rs1570360, rs3025039, and rs833061 played a key role in PDR etiology in Caucasians. rs2010963 was associated with increased risk of PDR in overall populations. A significant association between rs699947, rs3025039, and rs833061 and NPDR risk in overall populations and Asians was found. A significant association was observed between rs2010963 and increased NPDR risk in overall and Caucasian populations. This study provides a new insight into the parthenogenesis of diabetic retinopathy. Targeting VEGF SNPs may be a potential of therapeutic approach for the treatment of DR, PDR, and NPDR.

\section{Introduction}

Type 2 diabetes mellitus (T2DM) is a common metabolic disorder characterized by chronic elevation of blood glucose levels due to peripheral insulin resistance with deleterious effects on both micro- and macrocirculation [1, 2]. In 2011, the worldwide prevalence of T2DM was 366 million people, and is projected to rise to 552 million by 2030 $[3,4]$. More importantly, T2DM can incur high rates of complications,

Xiaorong Li and Juping Liu contributed equally to this work. morbidity, and mortality, thereby generating great socioeconomic burdens to both developing and developed countries [4].

Among the complications of T2DM, diabetic retinopathy (DR), including both proliferative diabetic retinopathy (PDR) and nonproliferative diabetic retinopathy (NPDR), has been recognized as a major burden on the health system. In addition, DR is a leading cause of blindness in developed countries [4-6]. Due to the high incidence of DR and its deleterious effects on patients' vision, special attention has been paid to explore the associated risk factors 
of this complication. The etiology and pathogenesis of both PDR and NPDR remain unclear as they involve multiple factors [6-10].

Vascular endothelial growth factor (VEGF) is a multifunctional cytokine that promotes angiogenesis and vascular permeability [1]. Under physiological conditions, VEGF is expressed at low levels in the eye [11]; under pathological circumstances, the expression of VEGF is upregulated, and VEGF overexpression promotes vessel endothelial cell proliferation, migration, tube formation, and sprouting, thereby subserving a contributing factor for DR [11]. Moreover, VEGF is also considered a primary initiator of PDR and a potential mediator of NPDR [2]. Hence VEGF gene and its polymorphic variants (single nucleotide polymorphisms, SNPs) play crucial roles in DR, characterized by impaired vascular permeability and neovascularization [12]. However, the association between VEGF gene polymorphisms and the susceptibility to DR, PDR, and NPDR has not been completely established [1, 5, 7, 11-33]. Porojan et al. [12] demonstrated that VEGF 936C/T polymorphism was a genetic risk factor for NPDR. Yuan et al. [11] found that among Chinese Han individuals with T2DM, polymorphism -634G/C of VEGF gene was not correlated with NPDR or PDR, which was consistent with the results shown by Nakamura et al. [15] and discrepant with those published by Yang et al. [7].

To determine whether VEGF SNPs are associated with the risk of DR, several meta-analyses have been performed, though the results varied. For example, the study by Xie et al. [34] showed that the SNPs rs3025039 and rs833061 were most likely associated with an increased risk of DR. On the other hand, no significant association was found between VEGF 2578C/A polymorphism (rs699947) and DR risk, which was consistent with the results of Gong et al. [35]. By contrast, the results from Wang et al. [36] supported the association between VEGF $2578 \mathrm{C} / \mathrm{A}$ polymorphism and the incidence of DR in Asian population, but not in Caucasian population. In addition, Zhao and colleagues. [37] confirmed the association between VEGF 634G/C polymorphism and the initiation of DR in the patents with T2DM. However, these findings were inconsistent with those published by Xie et al. [34]. The previous meta-analyses only analyzed the association between rs3025039, rs833061, rs2010963, and rs699947 with DR susceptibility. Whereas other VEGF gene polymorphisms, such as rs10434, rs1570360, rs25648, rs2146323, rs3025021, and rs13207351, are well known, yet their roles in the etiology and development of DR remain largely unknown. Although an increasing number of VEGF gene polymorphisms have been found associated with susceptibility to DR, PDR or NPDR [13, 23, 24, 28], no meta-analysis has been conducted to explore the associations between these novel VEGF gene polymorphisms and the risk of DR. The aim of the current meta-analysis is to explore the associations between the ten VEGF gene polymorphisms and the risks of DR, PDR, and NPDR.

\section{Materials and Methods}

\section{Literature search}

A systematic online search was conducted using 'PubMed', 'EMBASE', and 'the Cochrane Library' to identify the case-control studies regarding the relationship between VEGF gene polymorphisms and susceptibility to DR, PDR or NPDR. The following search terms were used to identify the eligible VEGF gene polymorphisms: ('diabetic retinopathy' OR 'DR' OR 'proliferative diabetic retinopathy' OR 'PDR' OR 'nonproliferative diabetic retinopathy' OR 'NPDR') AND ('VEGF' OR 'vascular endothelial growth factor') AND ('polymorphism' OR ‘single nucleotide polymorphism' OR 'SNP' OR ‘variation'). We found that VEGF 2578C/A (rs699947), VEGF 1612G/A (rs10434), VEGF 634(405)G/C (rs201096/rs2010963), VEGF 1154G/A (rs1570360), VEGF 936C/T (rs3025039), VEGF 1498C/T (rs833061), VEGF 7C/T (rs25648), VEGF 5092(6112)A/C (rs2146323), VEGF 9162(10180)C/T (rs3025021), VEGF 1190G/A (rs13207351) were analyzed in previous case-control studies and included into the meta-analysis. No language restrictions were applied. Unpublished literature search was conducted by looking into the reference lists from the selected studies, reviews, and conference reports.

\section{Inclusion and exclusion criteria}

The inclusion criteria of our meta-analysis were as follows: (1) case-control studies; (2) evaluation of DR, PDR or NPDR risk including the analysis from at least one identified VEGF gene polymorphisms; (3) detailed genotype frequency or numbers of alleles and genotypes between cases and controls. The exclusion criteria were: (1) reviews and case reports; (2) no available data; (3) duplicate reports.

\section{Data extraction}

Data from the eligible studies were extracted according to the inclusion and exclusion criteria by two authors (Yang $Q$ and Zhang Y) with further consensus reached. The following data were collected from each study: author list, year of publication, ethnicity, sample size of cases (DR, PDR and NPDR) and controls, VEGF gene polymorphisms, and HWE (Hardy-Weinberg Equilibrium).

\section{Data synthesis and statistical analysis}

We calculated odds ratios (OR) and $95 \%$ confidence interval $(\mathrm{Cl})$ to evaluate the association between VEGF gene polymorphisms and the risk of developing DR, PDR, and NPDR. An allele contrast model, heterozygote model, homozygote model, dominant, and recessive model were calculated to assess the associations between each VEGF gene polymorphism and the risk of DR, PDR and NPDR, respectively. Subgroup analysis was performed according to ethnicity and subtypes of DR. The heterogeneity of included studies was examined by a chi-squared-based $Q$ statistical test and quantified by $I^{2}$ metric value. If $\mathrm{I}^{2}$ value is $>50 \%$ or $\mathrm{p}<0.10$, the ORs were pooled by random effect model, otherwise, the fixed effect model was used. Sensitivity analysis was performed to assess the impact of each study on the present meta-analysis. In addition, the subgroup analysis was performed according to the ethnicity of the study populations. Stata 14.0 software (StataCorp, College Station, TX, USA) was used and a $p<0.05$ was considered as statistically significant.

\section{Results}

\section{Characteristics of included studies}

A total of 26 studies [ $1,5,7,11-33]$ containing 10 VEGF SNPs were finally collected and analyzed. The process of study selection and the inclusion process are shown in > Fig. 1. Ten SNPs of VEGF gene 


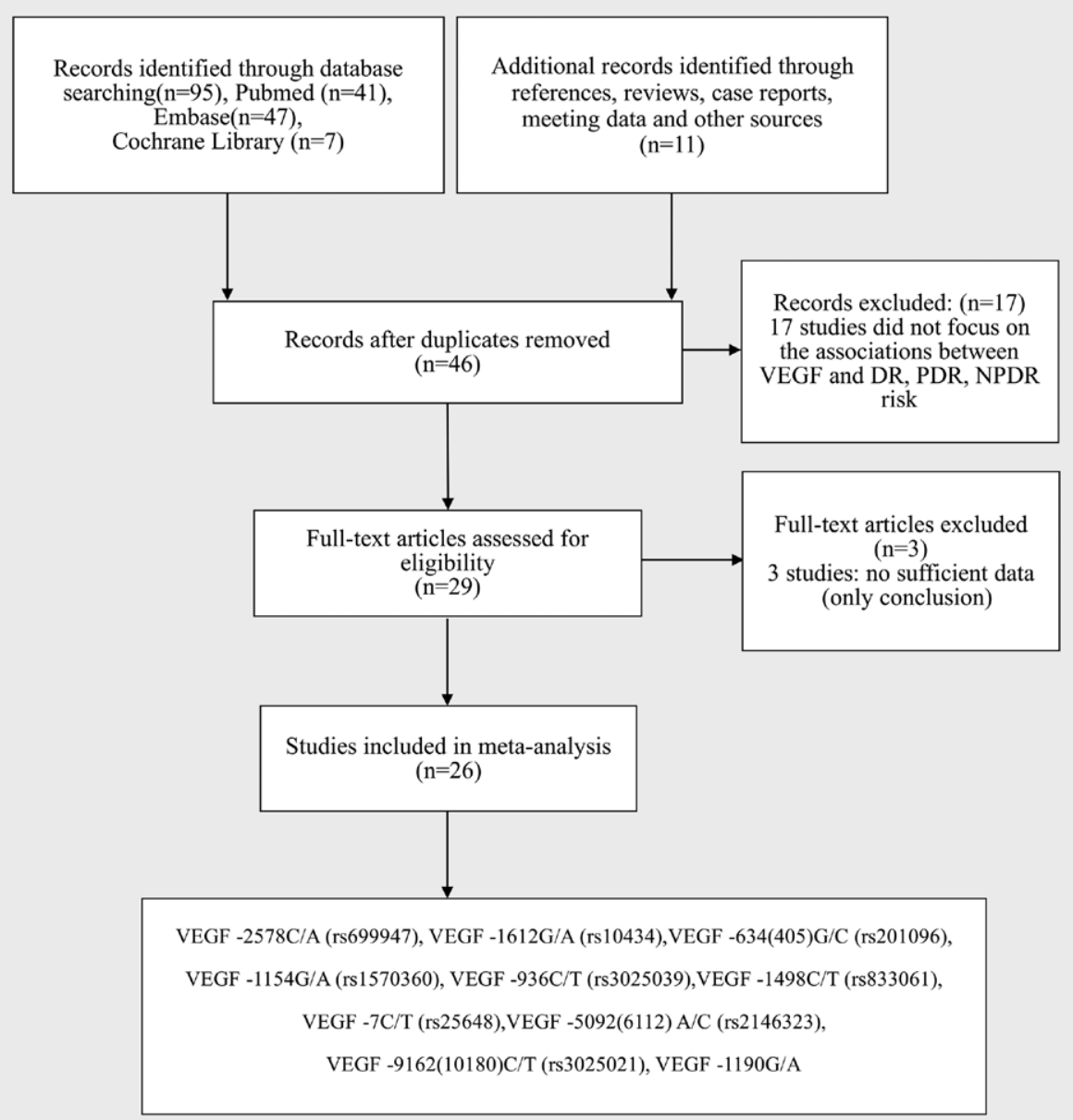

- Fig. 1 Flow chart showing the process of selection.

were analyzed, including VEGF 2578C/A (rs699947), VEGF 1612G/A (rs10434), VEGF 634(405)G/C (rs201096/rs2010963), VEGF 1154G/A (rs1570360), VEGF 936C/T (rs3025039), VEGF 1498C/T (rs833061), VEGF 7C/T (rs25648), VEGF 5092(6112)A/C (rs2146323), VEGF 9162(10180)C/T (rs3025021), and VEGF 1190G/A (rs13207351). All these studies [1, 5, 7, 11-33] had complied with HWE (Hardy-Weinberg Equilibrium). The general characteristics of the collected studies are summarized in $>$ Table 1.

\section{Meta-analysis}

VEGF gene polymorphisms and DR susceptibility

Our meta-analysis showed that VEGF 5092(6112)A/C (rs2146323), VEGF 9162(10180)C/T (rs3025021) and VEGF 1190G/A (rs13207351) were significantly associated with DR risk in either overall (rs2146323: CC vs. CA/AA: $O R=0.78,95 \% \mathrm{Cl}=0.62-0.9$, $p=0.027 ;$ rs3025021: TT vs. $C C: O R=0.46,95 \% C l=0.26-0.80$, $\mathrm{p}=0.006$; $\mathrm{TT}$ vs. TC/CC: $\mathrm{OR}=0.47,95 \% \mathrm{Cl}=0.27-0.80, \mathrm{p}=0.005$; rs13207351: A vs. $G: O R=1.52,95 \% C l=1.17-1.97, p=0.001 ; A A$ vs. $G G: O R=2.12,95 \% \mathrm{Cl}=1.25-3.61, \mathrm{p}=0.005 ; \mathrm{AA}$ vs. $\mathrm{AG} / \mathrm{GG}$ : $\mathrm{OR}=2.57,95 \% \mathrm{Cl}=1.59-4.17, \mathrm{p}<0.001$ ), Caucasians (rs2146323: CA vs. $A A: O R=1.74,95 \% C l=1.17-2.58, p=0.006 ; C C / C A$ vs. $A A$ : $O R=1.52,95 \% \mathrm{Cl}=1.05-2.22, \mathrm{p}=0.027 ; \mathrm{rs} 3025021: \mathrm{TT}$ vs. $\mathrm{CC}:$ $\mathrm{OR}=0.45,95 \% \mathrm{Cl}=0.24-0.84, \mathrm{p}=0.011$; $\mathrm{TT}$ vs. $\mathrm{TC} / \mathrm{CC}: \mathrm{OR}=0.43$,
$95 \% \mathrm{Cl}=0.24-0.77, \mathrm{p}=0.005 ; \mathrm{rs} 13207351:$ Avs. $\mathrm{G}: \mathrm{OR}=1.50,95 \%$ $\mathrm{Cl}=1.06-2.12, \mathrm{p}=0.021$; $\mathrm{AA}$ vs. $\mathrm{AG} / \mathrm{GG}: \mathrm{OR}=2.28,95 \% \mathrm{Cl}=1.03-$ 5.02, $p=0.041$ ), or Asians (rs2146323: C vs. $A: O R=0.63,95 \%$ $\mathrm{Cl}=0.42-0.94, \mathrm{p}=0.022 ; \mathrm{rs} 3025021$ : $\mathrm{T}$ vs. $\mathrm{C}: \mathrm{OR}=0.22,95 \%$ $\mathrm{Cl}=0.11-0.44, \mathrm{p}<0.001 ; \mathrm{TC}$ vs. $\mathrm{CC}: \mathrm{OR}=0.07,95 \% \mathrm{Cl}=0.02-0.23$, $\mathrm{p}<0.001 ; \mathrm{TT} / \mathrm{TC}$ vs. $\mathrm{CC}: \mathrm{OR}=0.14,95 \% \mathrm{Cl}=0.06-0.32, \mathrm{p}<0.001$; rs13207351: A vs. $G: O R=1.54,95 \% C l=1.04-2.27, p=0.029 ; A A$ vs. $G G: O R=4.07,95 \% \mathrm{Cl}=1.43-11.62, p=0.009 ; A A$ vs. $A G / G G$ : $\mathrm{OR}=3.95,95 \% \mathrm{Cl}=1.41-11.04, \mathrm{p}=0.009)$. Full data are shown in

- Tables 3 and 4.

Although no significant association was found between VEGF 7C/T (rs25648) and VEGF 1498 C/T (rs833061) and the risk of having DR in overall and Asian populations (both $\mathrm{p}>0.05$ ) ( $\triangleright$ Tables 2 , 3, and 4), rs25648 might increase the risk of DR in Caucasians (T vs. $\mathrm{C}: \mathrm{OR}=2.89,95 \% \mathrm{Cl}=1.43-5.83, \mathrm{p}=0.003$; $\mathrm{TC}$ vs. $\mathrm{CC}: \mathrm{OR}=4.12$, $95 \% \mathrm{Cl}=1.87-9.07, \mathrm{p}<0.001$; TT/TC vs. $C \mathrm{C}: \mathrm{OR}=3.71,95 \% \mathrm{Cl}=$ 1.73-7.94, $\mathrm{p}=0.001)$, and rs 833061 might decrease the $D R$ risk in Caucasians ( $T$ vs. C: $\mathrm{OR}=0.41,95 \% \mathrm{Cl}=0.18-0.90, \mathrm{p}=0.026$ ).

VEGF 634(405)G/C (rs201096/rs2010963) was significantly associated with increased risk of DR in overall (C vs. G: OR $=1.16,95 \%$ $\mathrm{Cl}=1.00-1.35, \mathrm{p}=0.049$; CC vs. $\mathrm{GG}: \mathrm{OR}=1.39,95 \% \mathrm{Cl}=1.12-1.73$, $p=0.003$; $G C$ vs. GG: $O R=1.18,95 \% C l=1.02-1.37, p=0.025 ; C C /$ GC vs. GG: $O R=1.22,95 \% \mathrm{Cl}=1.06-1.40, \mathrm{p}=0.004)$ and Caucasian 

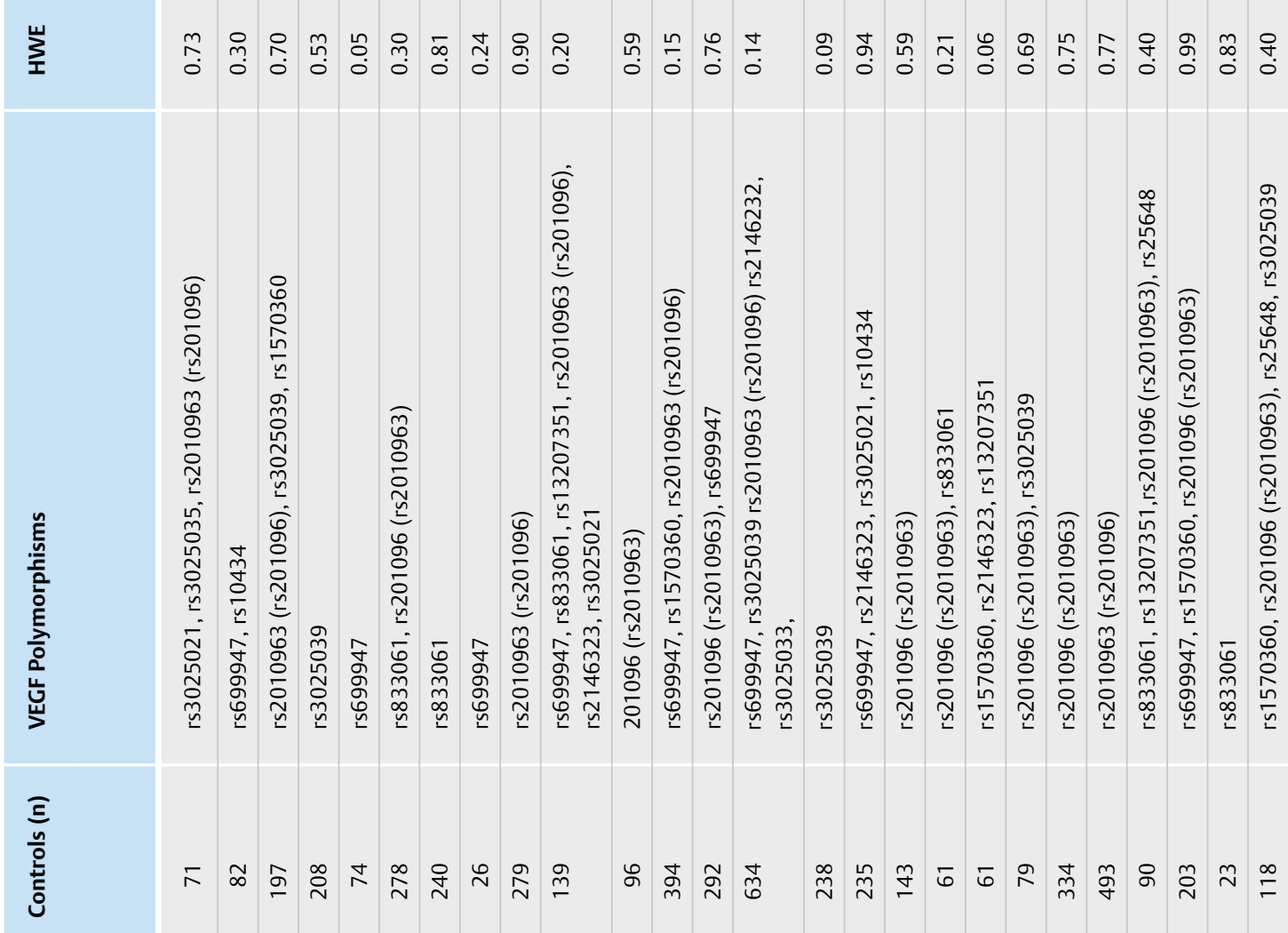

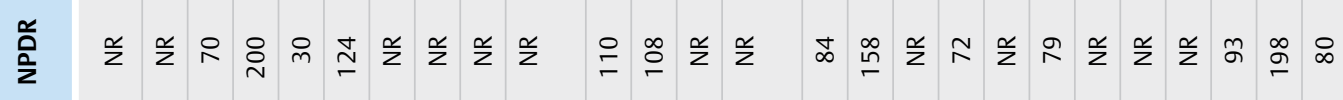

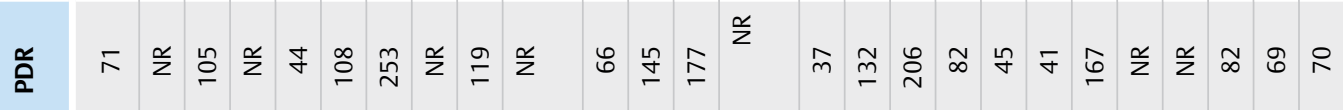

言

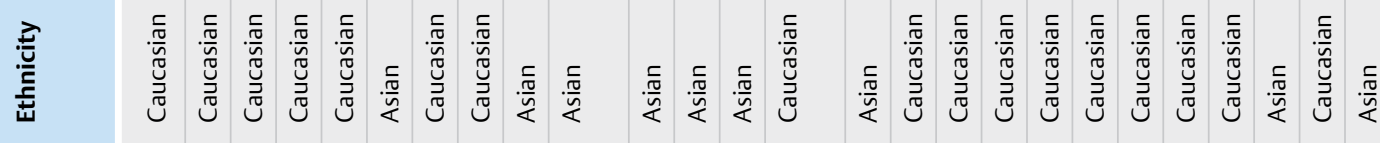

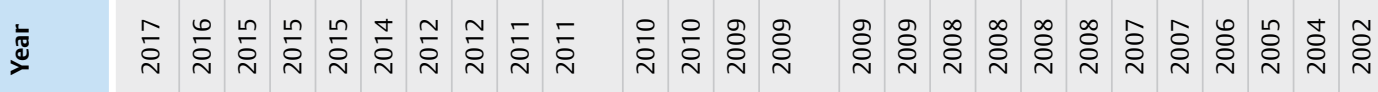

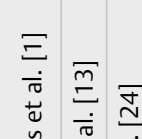

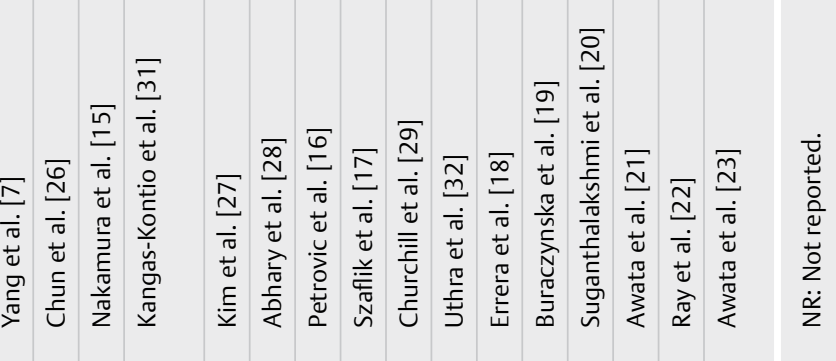


- Table 2 Results of associations between VEGF 2578C/A (rs699947), VEGF 1612G/A (rs10434), and VEGF 634(405)G/C (rs201096/ rs2010963) and risk of type 2 diabetic retinopathy.

\begin{tabular}{|c|c|c|c|c|c|c|}
\hline \multirow{2}{*}{ Genetic Models } & \multicolumn{3}{|c|}{ Test of association } & \multirow{2}{*}{ Model } & \multicolumn{2}{|c|}{ Test of heterogeneity } \\
\hline & OR & $95 \% \mathrm{Cl}$ & p-Value & & $\mathrm{I}^{2}(\%)$ & p-Value \\
\hline \multicolumn{7}{|c|}{ VEGF 2578C/A (rs699947) } \\
\hline \multicolumn{7}{|c|}{ Overall populations } \\
\hline A vs. C & 1.12 & $0.93-1.34$ & 0.231 & $\mathrm{R}$ & 50.2 & 0.041 \\
\hline AA vs. CC & 1.06 & $0.81-1.39$ & 0.669 & $\mathrm{~F}$ & 39.6 & 0.103 \\
\hline AC vs. CC & 1.27 & $0.94-1.71$ & 0.118 & $\mathrm{R}$ & 57.1 & 0.017 \\
\hline $\mathrm{AA} / \mathrm{AC}$ vs. CC & 1.25 & $0.94-1.65$ & 0.130 & $\mathrm{R}$ & 57.0 & 0.017 \\
\hline$A A$ vs. $A C / C C$ & 0.95 & $0.75-1.20$ & 0.667 & $\mathrm{~F}$ & 35.1 & 0.137 \\
\hline \multicolumn{7}{|l|}{ Caucasians } \\
\hline A vs. C & 1.04 & $0.88-1.22$ & 0.658 & $\mathrm{~F}$ & 0.0 & 0.815 \\
\hline AA vs. CC & 0.99 & $0.70-1.40$ & 0.085 & $\mathrm{~F}$ & 0.0 & 0.550 \\
\hline AC vs. CC & 1.27 & $0.97-1.68$ & 0.153 & $\mathrm{~F}$ & 26.6 & 0.235 \\
\hline AA/AC vs. CC & 1.21 & $0.93-1.57$ & 0.157 & $\mathrm{~F}$ & 15.6 & 0.314 \\
\hline AA vs. AC/CC & 0.90 & $0.68-1.18$ & 0.449 & $\mathrm{~F}$ & 0.0 & 0.539 \\
\hline \multicolumn{7}{|l|}{ Asians } \\
\hline A vs. C & 1.18 & $0.75-1.87$ & 0.469 & $\mathrm{R}$ & 84.0 & 0.002 \\
\hline AA vs. CC & 1.29 & $0.46-3.61$ & 0.628 & $\mathrm{R}$ & 77.9 & 0.011 \\
\hline AC vs. CC & 1.20 & $0.67-2.15$ & 0.549 & $\mathrm{R}$ & 83.1 & 0.003 \\
\hline $\mathrm{AA} / \mathrm{AC}$ vs. CC & 1.22 & $0.69-2.17$ & 0.498 & $\mathrm{R}$ & 83.9 & 0.002 \\
\hline$A A$ vs. $A C / C C$ & 1.20 & $0.46-3.09$ & 0.712 & $\mathrm{R}$ & 75.2 & 0.018 \\
\hline \multicolumn{7}{|c|}{ VEGF 1612G/A (rs10434) } \\
\hline \multicolumn{7}{|c|}{ Overall populations } \\
\hline A vs. G & 0.85 & $0.69-1.04$ & 0.118 & $\mathrm{~F}$ & 39.8 & 0.173 \\
\hline AA vs. GG & 0.71 & $0.35-1.45$ & 0.345 & $\mathrm{R}$ & 52.9 & 0.095 \\
\hline AG vs. GG & 1.07 & $0.78-1.46$ & 0.692 & $\mathrm{~F}$ & 0.0 & 0.946 \\
\hline AA/AG vs. GG & 0.94 & $0.70-1.27$ & 0.699 & $\mathrm{~F}$ & 0.0 & 0.689 \\
\hline AA vs. AG/GG & 0.70 & $0.35-1.39$ & 0.307 & $\mathrm{R}$ & 59.2 & 0.062 \\
\hline \multicolumn{7}{|l|}{ Caucasians } \\
\hline A vs. G & 0.87 & $0.60-1.24$ & 0.433 & $\mathrm{R}$ & 55.7 & 0.104 \\
\hline AA vs. GG & 0.71 & $0.29-1.75$ & 0.455 & $\mathrm{R}$ & 68.0 & 0.044 \\
\hline AG vs. GG & 1.06 & $0.74-1.53$ & 0.755 & $\mathrm{~F}$ & 0.0 & 0.831 \\
\hline AA/AG vs. GG & 0.91 & $0.65-1.29$ & 0.600 & $\mathrm{~F}$ & 0.0 & 0.516 \\
\hline AA vs. AG/GG & 0.69 & $0.29-1.63$ & 0.398 & $\mathrm{R}$ & 72.4 & 0.027 \\
\hline \multicolumn{7}{|l|}{ Asians } \\
\hline A vs. G & 1.00 & $0.60-1.66$ & 0.988 & $\mathrm{~F}$ & - & - \\
\hline AA vs. GG & 0.79 & $0.19-3.26$ & 0.748 & $\mathrm{R}$ & - & - \\
\hline AG vs. GG & 1.08 & $0.59-2.00$ & 0.803 & $\mathrm{~F}$ & - & - \\
\hline AA/AG vs. GG & 1.04 & $0.58-1.85$ & 0.902 & $\mathrm{~F}$ & - & - \\
\hline AA vs. AG/GG & 0.78 & $0.19-3.19$ & 0.730 & $\mathrm{R}$ & - & - \\
\hline \multicolumn{7}{|c|}{ VEGF 634(405)G/C (rs201096/rs2010963) } \\
\hline \multicolumn{7}{|c|}{ Overall populations } \\
\hline C vs. G & 1.16 & $1.00-1.35$ & 0.049 & $\mathrm{R}$ & 56.3 & 0.011 \\
\hline CC vs. GG & 1.39 & $1.12-1.73$ & 0.003 & $\mathrm{~F}$ & 48.1 & 0.037 \\
\hline GC vs. GG & 1.18 & $1.02-1.37$ & 0.025 & $\mathrm{~F}$ & 4.60 & 0.400 \\
\hline CC/GC vs. GG & 1.22 & $1.06-1.40$ & 0.004 & $\mathrm{~F}$ & 34.7 & 0.121 \\
\hline CC vs. GC/GG & 1.22 & $0.89-1.66$ & 0.214 & $\mathrm{R}$ & 57.9 & 0.008 \\
\hline
\end{tabular}


- Table 2 Continued

\begin{tabular}{|c|c|c|c|c|c|c|}
\hline \multirow{2}{*}{ Genetic Models } & \multicolumn{3}{|c|}{ Test of association } & \multirow{2}{*}{ Model } & \multicolumn{2}{|c|}{ Test of heterogeneity } \\
\hline & OR & $95 \% \mathrm{Cl}$ & p-Value & & $I^{2}(\%)$ & p-Value \\
\hline \multicolumn{7}{|l|}{ Caucasians } \\
\hline C vs. G & 1.19 & $1.04-1.37$ & 0.013 & $\mathrm{~F}$ & 47.5 & 0.090 \\
\hline CC vs. GG & 1.67 & $1.18-2.37$ & 0.004 & $\mathrm{~F}$ & 0.00 & 0.432 \\
\hline GC vs. GG & 1.20 & $0.98-1.48$ & 0.080 & $\mathrm{~F}$ & 0.00 & 0.441 \\
\hline CC/GC vs. GG & 1.27 & $1.04-1.54$ & 0.017 & $\mathrm{~F}$ & 8.70 & 0.360 \\
\hline CC vs. GC/GG & 1.33 & $0.77-2.27$ & 0.307 & $\mathrm{R}$ & 66.4 & 0.011 \\
\hline \multicolumn{7}{|l|}{ Asians } \\
\hline Cvs. G & 1.13 & $0.89-1.44$ & 0.321 & $\mathrm{R}$ & 69.0 & 0.012 \\
\hline CC vs. GG & 1.28 & $0.75-2.16$ & 0.362 & $\mathrm{R}$ & 68.9 & 0.012 \\
\hline GC vs. GG & 1.16 & $0.94-1.43$ & 0.156 & $\mathrm{~F}$ & 29.0 & 0.228 \\
\hline CC/GC vs. GG & 1.21 & $0.88-1.66$ & 0.236 & $\mathrm{R}$ & 58.3 & 0.048 \\
\hline CC vs. GC/GG & 1.14 & $0.78-1.67$ & 0.510 & $\mathrm{R}$ & 53.5 & 0.072 \\
\hline
\end{tabular}

populations ( $C$ vs. $\mathrm{G}$ : $\mathrm{OR}=1.19,95 \% \mathrm{Cl}=1.04-1.37, \mathrm{p}=0.013 ; \mathrm{CC}$ vs. $G G: O R=1.67,95 \% \mathrm{Cl}=1.18-2.37, p=0.004 ; C C / G C$ vs. GG: $\mathrm{OR}=1.27,95 \% \mathrm{Cl}=1.04-1.54, \mathrm{p}=0.017$ ), while no significant association was detected between these SNPs and DR risk in Asians $(p>0.05)$.

In addition, rs3025039 was associated with increased risk of DR in both overall (TT vs. CC: $\mathrm{OR}=3.26,95 \% \mathrm{Cl}=1.07-9.88, \mathrm{p}=0.037$; TT vs. TC/CC: $O R=2.42,95 \% \mathrm{Cl}=1.44-4.07, \mathrm{p}=0.001)$ and Asian populations ( $\mathrm{T}$ vs. $\mathrm{C}$ : $\mathrm{OR}=2.55,95 \% \mathrm{Cl}=1.10-5.93, \mathrm{p}=0.029$; $\mathrm{TT}$ vs. $C C$ : $O R=6.73,95 \% \mathrm{Cl}=1.74-26.06, p=0.006$; $T T$ vs. $T C / C C$ : $\mathrm{OR}=5.67,95 \% \mathrm{Cl}=1.47-21.90, \mathrm{p}=0.012$ ), while it might not play an important role in Caucasians.

Our analyses showed no significant association between VEGF 2578C/A (rs699947), VEGF 1612G/A (rs10434), and VEGF 1154G/A (rs1570360) and susceptibility to DR in overall, Caucasian, and Asian populations (both $p>0.05$ ) (

\section{VEGF Gene Polymorphisms and PDR Risk}

The rs1570360 SNP significantly increased the risk of PDR in overall (rs1570360: $A G$ vs. GG: $O R=1.42,95 \% \mathrm{Cl}=1.07-1.88, p=0.014$; $A A / A G$ vs. $G G: O R=1.65,95 \% C l=1.01-2.70, p=0.045)$ and $C a u-$ casian populations (rs1570360: AG vs. GG: $O R=1.79,95 \%$ $\mathrm{Cl}=1.12-2.85, \mathrm{p}=0.014)$; there was no significant association between this SNP and PDR risk in Asian populations (both $p>0.05$,

\section{- Table 5).}

The rs3025039 SNP was significantly associated with increased risk of PDR in overall (T vs. $C$ : $O R=2.38,95 \% C l=1.34-4.24, p=0.003$; TT vs. $C C:$ OR $=7.26,95 \% \mathrm{Cl}=3.65-14.44, \mathrm{p}<0.001$; $\mathrm{TC}$ vs. CC: $\mathrm{OR}=2.22,95 \% \mathrm{Cl}=1.06-4.64, \mathrm{p}=0.035 ; \mathrm{TT} / \mathrm{TC}$ vs. $\mathrm{CC}: \mathrm{OR}=2.53$, $95 \% \mathrm{Cl}=1.19-5.38, \mathrm{p}=0.016$; TT vs. TC/CC: $\mathrm{OR}=4.82,95 \%$ $\mathrm{Cl}=2.51-9.25, \mathrm{p}<0.001$ ), Caucasian (TT vs. $\mathrm{CC}: \mathrm{OR}=6.32,95 \%$ $\mathrm{Cl}=2.84-14.06$, $\mathrm{p}<0.001$; $\mathrm{TT}$ vs. $\mathrm{TC} / \mathrm{CC}: \mathrm{OR}=3.85$, $95 \% \mathrm{Cl}=1.81-$ $8.20, p<0.001$ ), and Asian populations (T vs. C: $O R=2.92,95 \%$
$\mathrm{Cl}=1.00-8.52, \mathrm{p}=0.050$; $\mathrm{TT}$ vs. $\mathrm{CC}: \mathrm{OR}=18.28,95 \% \mathrm{Cl}=1.15-$ 289.72, $\mathrm{p}=0.039$; $\mathrm{TT}$ vs. TC/CC: $\mathrm{OR}=9.21,95 \% \mathrm{Cl}=2.32-36.65$, $\mathrm{p}=0.002$ ).

The rs699947 SNP was significantly associated with increased risk of PDR in either overall ( $A$ vs. $C$ : $O R=1.34,95 \% \mathrm{Cl}=1.10-1.64$, $p=0.003 ; A C$ vs. $C C: O R=1.61,95 \% C l=1.23-2.10, p<0.001 ; A A /$ $A C$ vs. $C C: O R=1.57,95 \% C l=1.22-2.03, p<0.001)$ or Asian populations (A vs. $C$ : $O R=1.37,95 \% C l=1.11-1.69, p=0.004 ; A C$ vs. $C C: O R=1.57,95 \% \mathrm{Cl}=1.01-2.43, \mathrm{p}=0.044 ; \mathrm{AA} / \mathrm{AC}$ vs. $\mathrm{CC}$ : $\mathrm{OR}=1.55,95 \% \mathrm{Cl}=1.18-2.03, \mathrm{p}=0.001)$, while no significant association was found in Caucasians (all p >0.05) ( Table 5).

Significant association was found between rs833061 and decreased risk of PDR in Caucasians only (T vs. C: OR $=0.63,95 \%$ $\mathrm{Cl}=0.50-0.81, \mathrm{p}<0.001$; $\mathrm{TT}$ vs. $\mathrm{CC}: \mathrm{OR}=0.34,95 \% \mathrm{Cl}=0.17-0.69$, $\mathrm{p}=0.003 ; \mathrm{TT}$ vs. TC/CC: $\mathrm{OR}=0.63,95 \% \mathrm{Cl}=0.45-0.90, \mathrm{p}=0.010)$ ( Table 5).

Interestingly, rs201096 (rs2010963) was a risk contributor to PDR in overall populations (CC vs. GC/GG: OR $=1.26$, $95 \% \mathrm{Cl}=1.06-$ $1.50, p=0.008$ ), while no significant association was detected between rs201096 (rs2010963) and PDR risk in Asians and Caucasians, as indicated in > Table $\mathbf{5}$.

\section{VEGF Gene Polymorphisms and Risk of NPDR}

The rs699947 and rs833061 SNPs were significantly associated with increased susceptibility to NPDR in overall (rs699947: A vs. C: $\mathrm{OR}=1.42,95 \% \mathrm{Cl}=1.05-1.91, \mathrm{p}=0.021 ; \mathrm{AC}$ vs. $\mathrm{CC}: \mathrm{OR}=1.77,95 \%$ $\mathrm{Cl}=1.18-2.65, \mathrm{p}=0.005 ; \mathrm{AA} / \mathrm{AC}$ vs. $\mathrm{CC}: \mathrm{OR}=1.73,95 \% \mathrm{Cl}=1.17-$ 2.54, $\mathrm{p}=0.006$; $\mathrm{rs} 833061$ : $\mathrm{TT}$ vs. $\mathrm{CC}: \mathrm{OR}=2.14,95 \% \mathrm{Cl}=1.07-4.26$, $\mathrm{p}=0.031$; $\mathrm{TT}$ vs. $\mathrm{TC} / \mathrm{CC}: \mathrm{OR}=1.67,95 \% \mathrm{Cl}=1.12-2.48, \mathrm{p}=0.011$ ) and Asian populations (rs699947: $\mathrm{A}$ vs. $\mathrm{C}: \mathrm{OR}=1.55,95 \% \mathrm{Cl}=1.11-$ 2.17, $p=0.010$; $A C$ vs. $C C: O R=1.85,95 \% C l=1.18-2.91, p=0.007$; $A A / A C$ vs. $C C: O R=1.83,95 \% C l=1.18-2.91, p=0.006 ;$ rs833061: T vs. $C: O R=1.90,95 \% \mathrm{Cl}=1.30-2.77, \mathrm{p}=0.001 ; \mathrm{TT}$ vs. $\mathrm{CC}$ : 
- Table 3 Results of associations between VEGF 1154G/A (rs1570360), VEGF 936C/T (rs3025039), VEGF 7C/T (rs25648), and VEGF 5092 A/C (rs2146323) and risk of type 2 diabetic retinopathy.

\begin{tabular}{|c|c|c|c|c|c|c|}
\hline \multirow{2}{*}{ Genetic Models } & \multicolumn{3}{|c|}{ Test of association } & \multirow{2}{*}{ Model } & \multicolumn{2}{|c|}{ Test of heterogeneity } \\
\hline & OR & $95 \% \mathrm{Cl}$ & p-Value & & $I^{2}(\%)$ & p-Value \\
\hline \multicolumn{7}{|c|}{ VEGF 1154G/A (rs1570360) } \\
\hline \multicolumn{7}{|c|}{ Overall populations } \\
\hline A vs. G & 1.08 & $0.90-1.31$ & 0.393 & $\mathrm{~F}$ & 0.0 & 0.523 \\
\hline AA vs. GG & 1.12 & $0.64-1.97$ & 0.699 & $\mathrm{~F}$ & 0.0 & 0.460 \\
\hline AG vs. GG & 1.11 & $0.89-1.38$ & 0.375 & $\mathrm{~F}$ & 0.0 & 0.528 \\
\hline AA/AG vs. GG & 1.10 & $0.89-1.37$ & 0.366 & $\mathrm{~F}$ & 0.0 & 0.521 \\
\hline AA vs. AG/GG & 1.06 & $0.61-1.86$ & 0.838 & $\mathrm{~F}$ & 0.0 & 0.475 \\
\hline \multicolumn{7}{|l|}{ Caucasians } \\
\hline A vs. G & 1.32 & $0.93-1.89$ & 0.122 & $\mathrm{~F}$ & - & - \\
\hline AA vs. GG & 1.59 & $0.60-4.16$ & 0.348 & $\mathrm{~F}$ & - & - \\
\hline AG vs. GG & 1.36 & $0.87-2.12$ & 0.176 & $\mathrm{~F}$ & - & - \\
\hline AA/AG vs. GG & 1.39 & $0.91-2.12$ & 0.130 & $\mathrm{~F}$ & - & - \\
\hline AA vs. AG/GG & 1.43 & $0.55-3.71$ & 0.460 & $\mathrm{~F}$ & - & - \\
\hline \multicolumn{7}{|l|}{ Asians } \\
\hline A vs. G & 1.01 & $0.81-1.25$ & 0.960 & $\mathrm{~F}$ & 0.0 & 0.750 \\
\hline AA vs. GG & 0.93 & $0.46-1.88$ & 0.834 & $\mathrm{~F}$ & 0.0 & 0.412 \\
\hline AG vs. GG & 1.03 & $0.80-1.38$ & 0.810 & $\mathrm{~F}$ & 0.0 & 0.573 \\
\hline AA/AG vs. GG & 1.02 & $0.80-1.31$ & 0.872 & $\mathrm{~F}$ & 0.0 & 0.684 \\
\hline AA vs. AG/GG & 0.90 & $0.45-1.82$ & 0.772 & $\mathrm{~F}$ & 0.0 & 0.393 \\
\hline \multicolumn{7}{|c|}{ VEGF 936C/T (rs3025039) } \\
\hline \multicolumn{7}{|c|}{ Overall populations } \\
\hline T vs. C & 1.67 & $0.96-2.91$ & 0.071 & $\mathrm{R}$ & 88.5 & $<0.001$ \\
\hline TT vs. CC & 3.26 & $1.07-9.88$ & 0.037 & $\mathrm{R}$ & 61.0 & 0.053 \\
\hline TC vs. CC & 1.74 & $0.89-3.42$ & 0.108 & $\mathrm{R}$ & 88.7 & $<0.001$ \\
\hline TT/TC vs. CC & 1.82 & $0.91-3.64$ & 0.088 & $\mathrm{R}$ & 89.8 & $<0.001$ \\
\hline TT vs. TC/CC & 2.42 & $1.44-4.07$ & 0.001 & $\mathrm{~F}$ & 45.1 & 0.141 \\
\hline \multicolumn{7}{|l|}{ Caucasians } \\
\hline T vs. C & 1.24 & $0.59-2.64$ & 0.569 & $\mathrm{R}$ & 89.1 & $<0.001$ \\
\hline TT vs. CC & 1.97 & $0.38-10.08$ & 0.418 & $\mathrm{R}$ & 80.6 & 0.023 \\
\hline TC vs. CC & 1.31 & $0.57-3.01$ & 0.519 & $\mathrm{R}$ & 87.0 & $<0.001$ \\
\hline TT/TC vs. CC & 1.33 & $0.54-3.27$ & 0.530 & $\mathrm{R}$ & 89.6 & $<0.001$ \\
\hline TT vs. TC/CC & 1.60 & $0.48-5.35$ & 0.446 & $\mathrm{R}$ & 66.1 & 0.086 \\
\hline \multicolumn{7}{|l|}{ Asians } \\
\hline T vs. C & 2.55 & $1.10-5.93$ & 0.029 & $\mathrm{R}$ & 87.2 & 0.005 \\
\hline TT vs. CC & 6.73 & $1.74-26.06$ & 0.006 & $\mathrm{~F}$ & 12.8 & 0.284 \\
\hline TC vs. CC & 2.65 & $0.75-9.32$ & 0.129 & $\mathrm{R}$ & 91.8 & $<0.001$ \\
\hline TT/TC vs. CC & 2.89 & $0.89-9.37$ & 0.077 & $\mathrm{R}$ & 91.1 & 0.001 \\
\hline TT vs. TC/CC & 5.67 & $1.47-21.90$ & 0.012 & $\mathrm{~F}$ & 0.0 & 0.417 \\
\hline \multicolumn{7}{|c|}{ VEGF 7C/T (rs25648) } \\
\hline \multicolumn{7}{|c|}{ Overall populations } \\
\hline T vs. C & 1.48 & $0.42-5.19$ & 0.536 & $\mathrm{R}$ & 89.0 & 0.003 \\
\hline TT vs. CC & 0.42 & $0.11-1.58$ & 0.200 & $\mathrm{~F}$ & 0.0 & 0.861 \\
\hline TC vs. CC & 1.86 & $0.41-8.39$ & 0.418 & $\mathrm{R}$ & 90.0 & 0.002 \\
\hline TT/TC vs. CC & 1.71 & $0.40-7.40$ & 0.471 & $\mathrm{R}$ & 90.1 & 0.001 \\
\hline TT vs. TC/CC & 0.41 & $0.11-1.53$ & 0.185 & $\mathrm{~F}$ & 0.0 & 0.729 \\
\hline
\end{tabular}


$\checkmark$ Table 3 Continued

\begin{tabular}{|c|c|c|c|c|c|c|}
\hline \multirow{2}{*}{ Genetic Models } & \multicolumn{3}{|c|}{ Test of association } & \multirow{2}{*}{ Model } & \multicolumn{2}{|c|}{ Test of heterogeneity } \\
\hline & OR & $95 \% \mathrm{Cl}$ & p-Value & & $I^{2}(\%)$ & p-Value \\
\hline \multicolumn{7}{|l|}{ Caucasians } \\
\hline T vs. C & 2.89 & $1.43-5.83$ & 0.003 & $\mathrm{R}$ & - & - \\
\hline TT vs. CC & 0.33 & $0.01-8.10$ & 0.494 & $\mathrm{~F}$ & - & - \\
\hline TC vs. CC & 4.12 & $1.87-9.07$ & $<0.001$ & $\mathrm{R}$ & - & - \\
\hline $\mathrm{TT} / \mathrm{TC}$ vs. CC & 3.71 & $1.73-7.94$ & 0.001 & $\mathrm{R}$ & - & - \\
\hline TT vs. TC/CC & 0.25 & $0.01-6.15$ & 0.394 & $\mathrm{~F}$ & - & - \\
\hline \multicolumn{7}{|l|}{ Asians } \\
\hline T vs. C & 0.81 & $0.52-1.27$ & 0.352 & $\mathrm{R}$ & - & - \\
\hline TT vs. CC & 0.45 & $0.10-1.92$ & 0.278 & $\mathrm{~F}$ & - & - \\
\hline TC vs. CC & 0.89 & $0.52-1.52$ & 0.674 & $\mathrm{R}$ & - & - \\
\hline TT/TC vs. CC & 0.84 & $0.50-1.40$ & 0.496 & $R$ & - & - \\
\hline TT vs. TC/CC & 0.46 & $0.11-1.97$ & 0.296 & $\mathrm{~F}$ & - & - \\
\hline \multicolumn{7}{|c|}{ VEGF 5092(6112)A/C (rs2146323) } \\
\hline \multicolumn{7}{|c|}{ Overall populations } \\
\hline Cvs. A & 0.92 & $0.70-1.21$ & 0.545 & $\mathrm{R}$ & 59.2 & 0.044 \\
\hline CC vs. AA & 0.97 & $0.51-1.84$ & 0.928 & $\mathrm{R}$ & 56.9 & 0.054 \\
\hline CA vs. AA & 1.42 & $0.76-2.64$ & 0.276 & $\mathrm{R}$ & 55.4 & 0.062 \\
\hline CC/CA vs. AA & 1.17 & $0.63-2.18$ & 0.617 & $\mathrm{R}$ & 58.7 & 0.046 \\
\hline CC vs. CA/AA & 0.78 & $0.62-0.97$ & 0.027 & $\mathrm{~F}$ & 23.1 & 0.267 \\
\hline \multicolumn{7}{|l|}{ Caucasians } \\
\hline C vs. A & 1.01 & $0.84-1.21$ & 0.946 & $\mathrm{~F}$ & 44.3 & 0.146 \\
\hline CC vs. AA & 1.30 & $0.87-1.95$ & 0.197 & $\mathrm{~F}$ & 40.3 & 0.170 \\
\hline CA vs. AA & 1.74 & $1.17-2.58$ & 0.006 & $\mathrm{~F}$ & 45.6 & 0.137 \\
\hline CC/CA vs. AA & 1.52 & $1.05-2.22$ & 0.027 & $\mathrm{~F}$ & 39.8 & 0.173 \\
\hline CC vs. CA/AA & 0.82 & $0.64-1.06$ & 0.131 & $\mathrm{~F}$ & 29.1 & 0.237 \\
\hline \multicolumn{7}{|l|}{ Asians } \\
\hline Cvs. A & 0.63 & $0.42-0.94$ & 0.022 & $\mathrm{R}$ & - & - \\
\hline CC vs. AA & 0.41 & $0.16-1.01$ & 0.054 & $R$ & - & - \\
\hline CA vs. $A A$ & 0.58 & $0.22-1.52$ & 0.270 & $R$ & - & - \\
\hline CC/CA vs. AA & 0.46 & $0.19-1.13$ & 0.092 & $\mathrm{R}$ & - & - \\
\hline CC vs. CA/AA & 0.62 & $0.38-1.02$ & 0.060 & $\mathrm{~F}$ & - & - \\
\hline
\end{tabular}

$\mathrm{OR}=4.10,95 \% \mathrm{Cl}=1.40-12.06, \mathrm{p}=0.010 ; \mathrm{TT} / \mathrm{TC}$ vs. $\mathrm{CC}: \mathrm{OR}=3.49$, $95 \% \mathrm{Cl}=1.20-10.16, \mathrm{p}=0.022$; TT vs. TC/CC: $\mathrm{OR}=1.88,95 \%$ $\mathrm{Cl}=1.20-2.94, \mathrm{p}=0.006)$, while no significant association was found in Caucasians (all p >0.05) (

The rs3025039 SNP contributed significantly to the increased risk of NPDR in overall ( $\mathrm{T}$ vs. $\mathrm{C}: \mathrm{OR}=1.82,95 \% \mathrm{Cl}=1.13-2.92$, $\mathrm{p}=0.013$; $\mathrm{TT}$ vs. $\mathrm{CC}: \mathrm{OR}=3.49,95 \% \mathrm{Cl}=1.85-6.59, \mathrm{p}<0.001$; $\mathrm{TC}$ vs. $C C: O R=1.94,95 \% \mathrm{Cl}=1.04-3.62, p=0.036 ; \mathrm{TT} / \mathrm{TC}$ vs. $\mathrm{CC}$ : $\mathrm{OR}=2.03,95 \% \mathrm{Cl}=1.12-3.68, \mathrm{p}=0.020 ; \mathrm{TT}$ vs. $\mathrm{TC} / \mathrm{CC}: \mathrm{OR}=2.65$, $95 \% \mathrm{Cl}=1.43-4.89, \mathrm{p}=0.002$ ), Caucasian (TT vs. $\mathrm{CC}: \mathrm{OR}=3.12$, $95 \% \mathrm{Cl}=1.09-8.94, \mathrm{p}=0.034$ ), and Asian populations ( $\mathrm{T}$ vs. $\mathrm{C}$ : $\mathrm{OR}=2.33,95 \% \mathrm{Cl}=1.03-5.28, \mathrm{p}=0.043)$.
The rs201096 (rs2010963) SNP was significantly associated with an increased risk of NPDR in overall ( $C$ vs. G: OR $=1.23,95 \%$ $\mathrm{Cl}=1.04-1.45, \mathrm{p}=0.017$; CC vs. GC/GG: $\mathrm{OR}=1.42$, $95 \% \mathrm{Cl}=1.03-$ $1.98, p=0.034$ ) and Caucasian populations (Cvs. G: $O R=1.36,95 \%$ $\mathrm{Cl}=1.03-1.80, \mathrm{p}=0.029$; $\mathrm{CC}$ vs. $\mathrm{GC} / \mathrm{GG}$ : $\mathrm{OR}=2.15$, $95 \% \mathrm{Cl}=1.17-$ 3.93, $p=0.013$ ), while no significant association was found in Asians (all p >0.05, > Table 5).

No significant association was found between rs 1570360 and susceptibility to NPDR in overall, Asian, and Caucasian populations (both p >0.05) ( $\triangleright$ Table 5). 
- Table 4 Results of associations between VEGF 9162C/T (rs3025021), VEGF 1190G/A (rs13207351), and VEGF 1498 C/T (rs833061) and risk of type 2 diabetic retinopathy.

\begin{tabular}{|c|c|c|c|c|c|c|}
\hline \multirow{2}{*}{ Genetic Models } & \multicolumn{3}{|c|}{ Test of association } & \multirow{2}{*}{ Model } & \multicolumn{2}{|c|}{ Test of heterogeneity } \\
\hline & OR & $95 \% \mathrm{Cl}$ & p-Value & & $\mathbf{I}^{2}(\%)$ & p-Value \\
\hline \multicolumn{7}{|c|}{ VEGF $9162(10180) C / T($ rs3025021) } \\
\hline \multicolumn{7}{|c|}{ Overall populations } \\
\hline T vs. C & 0.67 & $0.39-1.15$ & 0.145 & $\mathrm{R}$ & 80.8 & 0.001 \\
\hline TT vs. CC & 0.46 & $0.26-0.80$ & 0.006 & $\mathrm{~F}$ & 0.0 & 0.531 \\
\hline TC vs. CC & 0.69 & $0.28-1.69$ & 0.412 & $\mathrm{R}$ & 86.4 & $<0.001$ \\
\hline TT/TC vs. CC & 0.67 & $0.30-1.47$ & 0.316 & $\mathrm{R}$ & 85.5 & $<0.001$ \\
\hline TT vs. TC/CC & 0.47 & $0.27-0.80$ & 0.005 & $\mathrm{~F}$ & 1.7 & 0.384 \\
\hline \multicolumn{7}{|l|}{ Caucasians } \\
\hline T vs. C & 0.87 & $0.69-1.10$ & 0.240 & $\mathrm{~F}$ & 0.0 & 0.454 \\
\hline TT vs. CC & 0.45 & $0.24-0.84$ & 0.011 & $\mathrm{~F}$ & 8.6 & 0.335 \\
\hline TC vs. CC & 1.19 & $0.86-1.64$ & 0.288 & $\mathrm{~F}$ & 0.0 & 0.719 \\
\hline TT/TC vs. CC & 1.02 & $0.75-1.39$ & 0.902 & $\mathrm{~F}$ & 0.0 & 0.626 \\
\hline TT vs. TC/CC & 0.43 & $0.24-0.77$ & 0.005 & $\mathrm{~F}$ & 23.8 & 0.269 \\
\hline \multicolumn{7}{|l|}{ Asians } \\
\hline T vs. C & 0.22 & $0.11-0.44$ & $<0.001$ & $\mathrm{R}$ & - & - \\
\hline TT vs. CC & 0.51 & $0.14-1.80$ & 0.294 & $\mathrm{~F}$ & - & - \\
\hline TC vs. CC & 0.07 & $0.02-0.23$ & $<0.001$ & $\mathrm{R}$ & - & - \\
\hline TT/TC vs. CC & 0.14 & $0.06-0.32$ & $<0.001$ & $\mathrm{R}$ & - & - \\
\hline TT vs. TC/CC & 0.71 & $0.20-2.49$ & 0.591 & $\mathrm{~F}$ & - & - \\
\hline \multicolumn{7}{|c|}{ VEGF 1190G/A (rs13207351) } \\
\hline \multicolumn{7}{|c|}{ Overall populations } \\
\hline A vs. G & 1.52 & $1.17-1.97$ & 0.001 & $\mathrm{~F}$ & 0.0 & 0.662 \\
\hline AA vs. GG & 2.12 & $1.25-3.61$ & 0.005 & $\mathrm{~F}$ & 7.8 & 0.338 \\
\hline AG vs. GG & 0.87 & $0.48-1.58$ & 0.642 & $\mathrm{R}$ & 51.2 & 0.129 \\
\hline AA/AG vs. GG & 1.26 & $0.89-1.77$ & 0.192 & $\mathrm{~F}$ & 0.0 & 0.744 \\
\hline AA vs. AG/GG & 2.57 & $1.59-4.17$ & $<0.001$ & $\mathrm{~F}$ & 31.6 & 0.232 \\
\hline \multicolumn{7}{|l|}{ Caucasians } \\
\hline A vs. G & 1.50 & $1.06-2.12$ & 0.021 & $\mathrm{~F}$ & 0.0 & 0.366 \\
\hline AA vs. GG & 1.63 & $0.87-3.05$ & 0.128 & $\mathrm{~F}$ & 0.0 & 0.934 \\
\hline AG vs. GG & 0.64 & $0.17-2.33$ & 0.495 & $\mathrm{R}$ & 72.7 & 0.056 \\
\hline AA/AG vs. GG & 1.17 & $0.72-1.90$ & 0.518 & $\mathrm{~F}$ & 0.0 & 0.509 \\
\hline AA vs. AG/GG & 2.28 & $1.03-5.02$ & 0.041 & $\mathrm{R}$ & 50.9 & 0.154 \\
\hline \multicolumn{7}{|l|}{ Asians } \\
\hline A vs. G & 1.54 & $1.04-2.27$ & 0.029 & $\mathrm{~F}$ & - & - \\
\hline AA vs. GG & 4.07 & $1.43-11.62$ & 0.009 & $\mathrm{~F}$ & - & - \\
\hline AG vs. GG & 1.08 & $0.64-1.81$ & 0.771 & $\mathrm{R}$ & - & - \\
\hline AA/AG vs. GG & 1.35 & $0.83-2.19$ & 0.230 & $\mathrm{~F}$ & - & - \\
\hline AA vs. AG/GG & 3.95 & $1.41-11.04$ & 0.009 & $\mathrm{~F}$ & - & - \\
\hline \multicolumn{7}{|c|}{ VEGF 1498 C/T (rs833061) } \\
\hline \multicolumn{7}{|c|}{ Overall populations } \\
\hline T vs. C & 0.86 & $0.17-4.23$ & 0.853 & $\mathrm{R}$ & 98.6 & $<0.001$ \\
\hline TT vs. CC & 0.64 & $0.22-1.87$ & 0.419 & $\mathrm{R}$ & 72.5 & 0.006 \\
\hline TC vs. CC & 0.90 & $0.61-1.32$ & 0.587 & $\mathrm{~F}$ & 46.0 & 0.116 \\
\hline TT/TC vs. CC & 0.80 & $0.38-1.66$ & 0.548 & $\mathrm{R}$ & 65.4 & 0.021 \\
\hline TT vs. TC/CC & 0.68 & $0.31-1.52$ & 0.347 & $\mathrm{R}$ & 87.7 & $<0.001$ \\
\hline
\end{tabular}


- Table 4 Continued

\begin{tabular}{|c|c|c|c|c|c|c|}
\hline \multirow{2}{*}{ Genetic Models } & \multicolumn{3}{|c|}{ Test of association } & \multirow{2}{*}{ Model } & \multicolumn{2}{|c|}{ Test of heterogeneity } \\
\hline & OR & $95 \% \mathrm{Cl}$ & p-Value & & $I^{2}(\%)$ & p-Value \\
\hline \multicolumn{7}{|l|}{ Caucasians } \\
\hline T vs. C & 0.41 & $0.18-0.90$ & 0.026 & $\mathrm{R}$ & 86.5 & 0.001 \\
\hline TT vs. CC & 0.57 & $0.24-1.36$ & 0.206 & $\mathrm{~F}$ & 6.3 & 0.344 \\
\hline TC vs. CC & 0.90 & $0.54-1.53$ & 0.707 & $\mathrm{~F}$ & 0.0 & 0.851 \\
\hline TT/TC vs. CC & 0.85 & $0.51-1.42$ & 0.538 & $\mathrm{~F}$ & 0.0 & 0.579 \\
\hline TT vs. TC/CC & 0.47 & $0.15-1.46$ & 0.191 & $\mathrm{R}$ & 75.9 & 0.016 \\
\hline \multicolumn{7}{|l|}{ Asians } \\
\hline T vs. C & 2.55 & $0.18-36.74$ & 0.491 & $\mathrm{R}$ & 99.2 & $<0.001$ \\
\hline TT vs. CC & 0.77 & $0.09-6.44$ & 0.809 & $\mathrm{R}$ & 91.2 & 0.001 \\
\hline TC vs. CC & 0.70 & $0.12-3.93$ & 0.685 & $\mathrm{R}$ & 85.9 & 0.008 \\
\hline TT/TC vs. CC & 0.74 & $0.10-5.39$ & 0.770 & $\mathrm{R}$ & 90.2 & 0.001 \\
\hline TT vs. TC/CC & 1.07 & $0.54-2.12$ & 0.849 & $\mathrm{R}$ & 80.8 & 0.023 \\
\hline
\end{tabular}

\section{Sensitivity analysis and publication bias}

A leave-one-out analysis was performed to estimate the sensitivity of the current meta-analysis. Any single study could be omitted, without any effect on the overall statistical significance, indicating that the results are stable. Funnel plot shape is symmetrical, therefore no publication bias in this study is shown (> Fig. 2).

\section{Discussion}

Abnormally increased VEGF concentrations were detected in patients with DR, which is characterized by neuronal and vascular dysfunction in retina at early stages with subsequent neovascularization and visual damage $[38,39]$. Likewise, VEGF overexpression in retina was found in the animal models of diabetes [38]. Furthermore, VEGF injection into the vitreous of non-human primates induces lesions characteristic of DR [40]. These findings pointed out that VEGF is a major contributor to DR, which could also be considered as a potential target for DR treatment.

Anti-VEGF injections have been an effective therapy to improve both vision and Diabetic Retinopathy Severity Scale score in DR patients $[38,41]$. It is considered as the standard treatment of diabetic macular edema [38]. Despite the improved visual outcomes of patients with DR treated with anti-VEGF agents, the unresponsiveness to the anti-VEGFs has been reported previously. Genetic polymorphisms appear to be another variable to analyze when the anti-VEGF therapy is ineffective [42]. In addition, VEGF protein expression has been shown to be influenced by genetic variations at VEGF gene locus, and the increased transcript levels of VEGF in the vitreous fluid promoted the development and progression of DR [36]. Therefore, studies indicate that the VEGF gene polymorphisms play a major role in DR etiology and pathophysiology [1, 5, 7, 11-33], yet these results were controversial.

\section{VEGF Gene Polymorphisms and DR Risk}

VEGF634(405)G/C (rs201096/rs2010963) promoter polymorphism is associated with an increased transcriptional and translational activities of VEGF gene, which may be responsible for the development of DR $[21,23]$. This study indicates that VEGF $634(405) G / C$ (rs201096/rs2010963) is significantly associated with the increased susceptibility of DR in two populations, overall and Caucasian. These results are consistent with those published by Qiu [43]. On the contrary, numerous significant differences were observed among Qiu's results [43]. The meta-analysis[43] was conducted to determine the association between VEGF 634G/C and DR risk. They included the studies of Petrovic et al. [16] and Nakamura et al. [15], however, these two studies sought to find the association between the $634 \mathrm{C} / \mathrm{G}$ polymorphism of VEGF gene and PDR. Therefore, it is not suitable to include these two studies, as they may have overestimated the SNP's impact on the DR risk. By contrast, a previous study conducted by Zhao et al. [37] did not support the association of VEGF 634 C/G polymorphism with either DR or PDR. The discrepancy is originated from two points: one study was included in the current meta-analysis [7], but was not in Zhao's [37]; moreover, the data in Zhao's study were analyzed inaccurately [15, 16, 31]. However, the meta-analyses conducted by us and others all indicate that no significant association was found between the SNP rs201096/rs2010963 and DR risk in Asians. Ethnicity and genetic background might play a predominant role. Many factors could determine the differences in the findings about Asian and Caucasian populations, such as sample size, study design, retinopathy grading scales, and genotyping techniques. Since sunlight exposure is a known risk factor of age-related macular degeneration, the increased exposure to sunlight in Caucasian areas could be another reason for DR [36].

VEGF 5092(6112)A/C (rs2146323) protects against DR in overall and Asian populations. However, significant association was ob- 


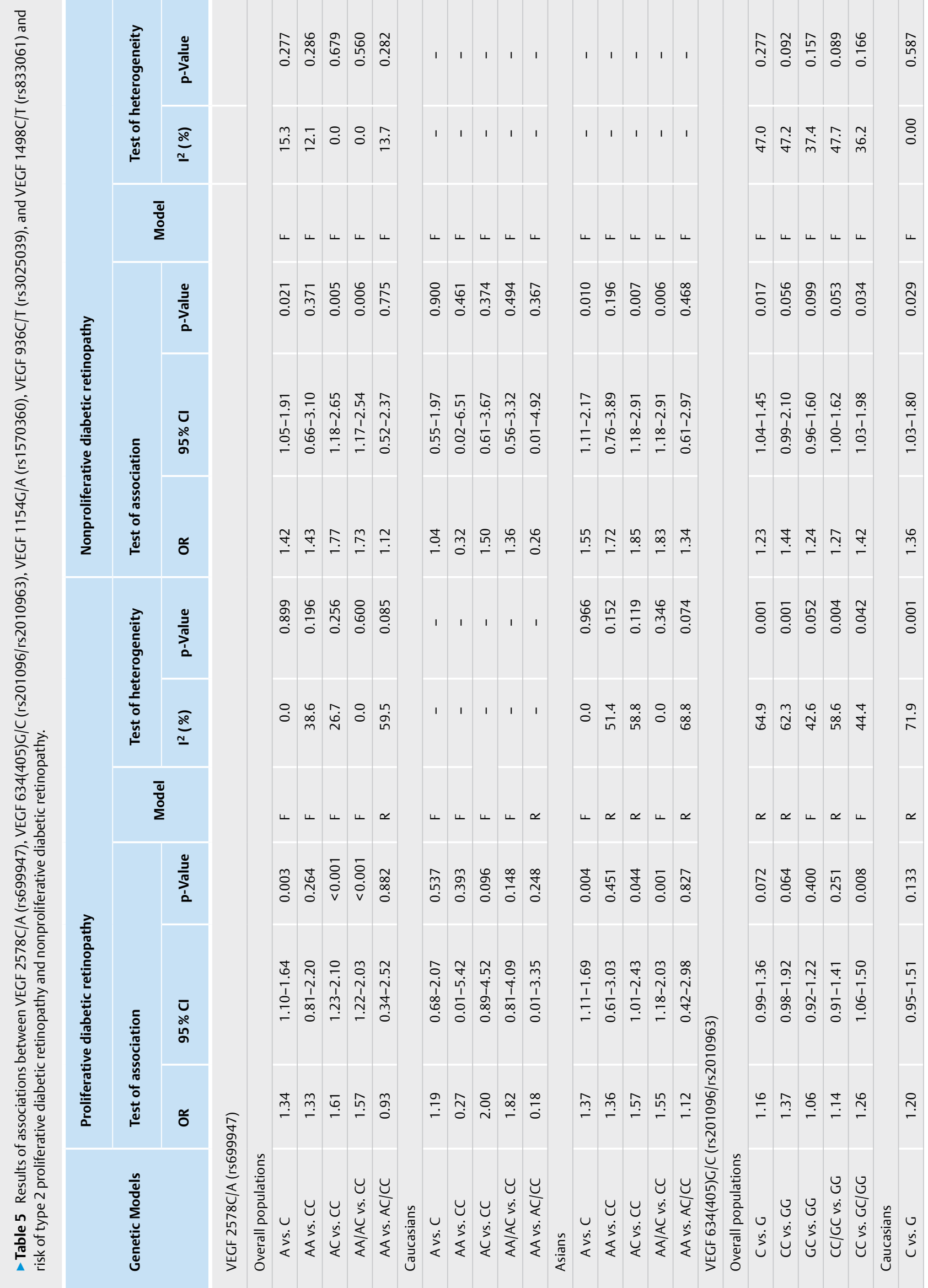




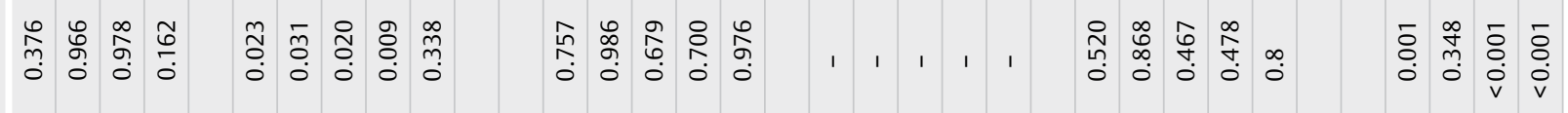

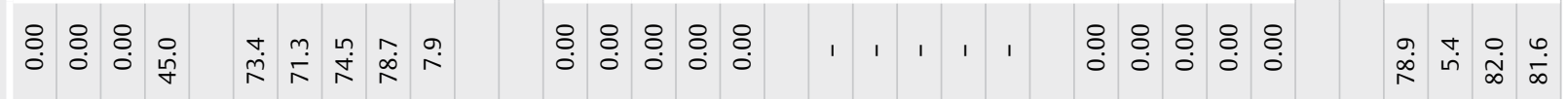

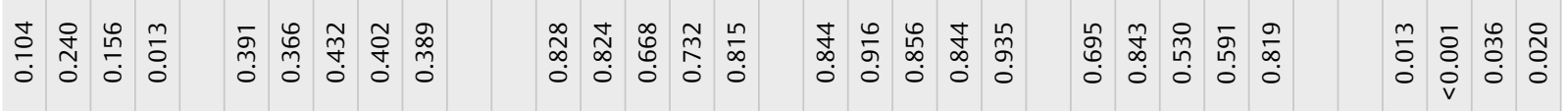

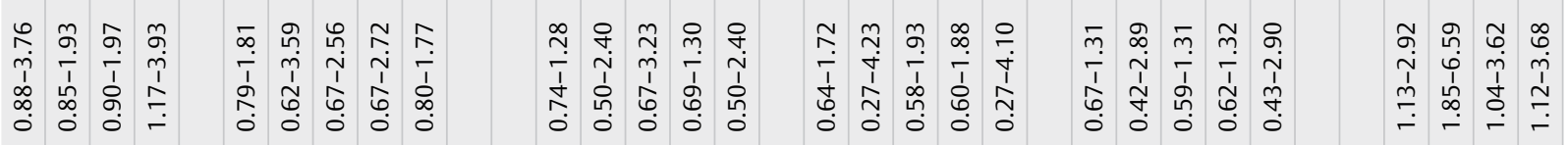

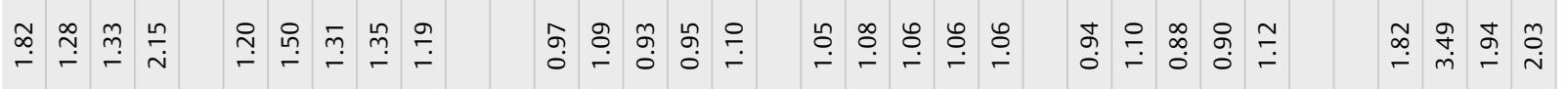

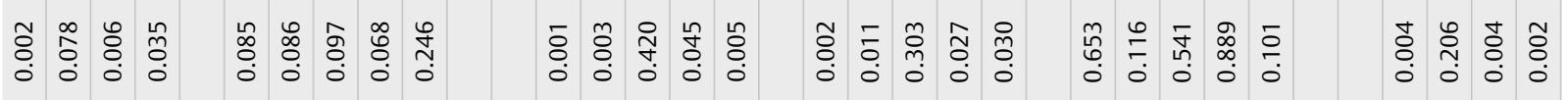

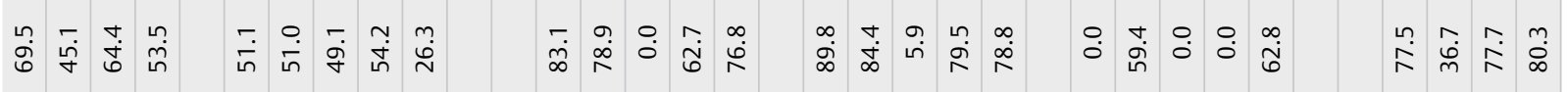

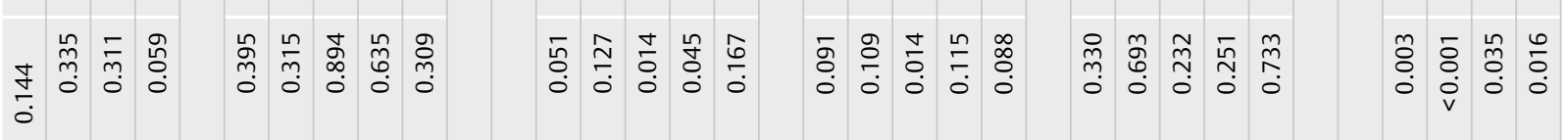

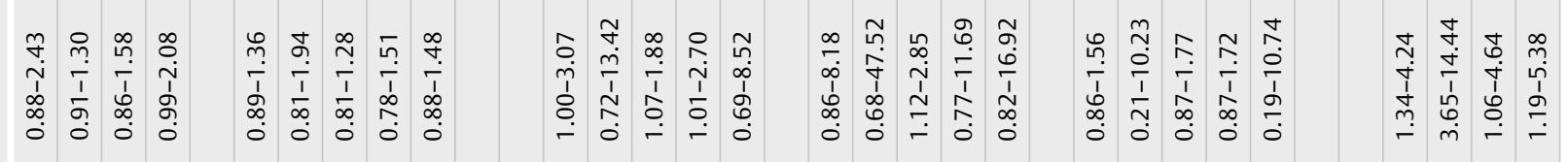

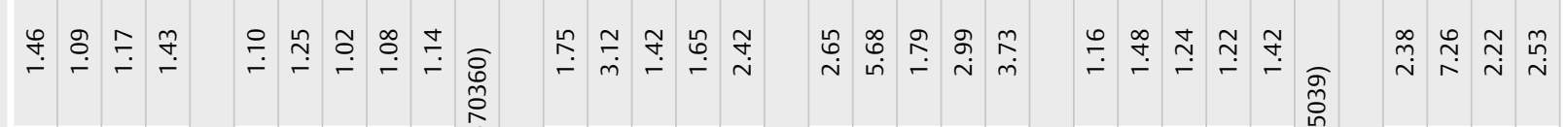

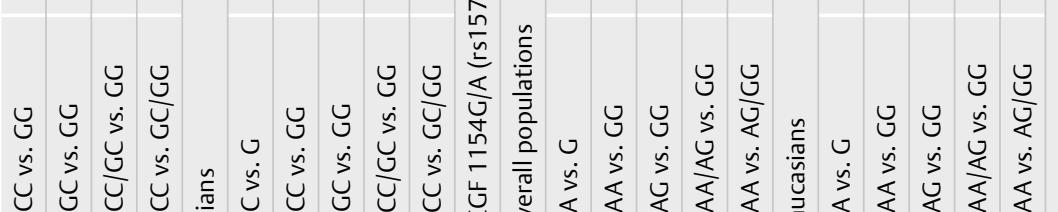

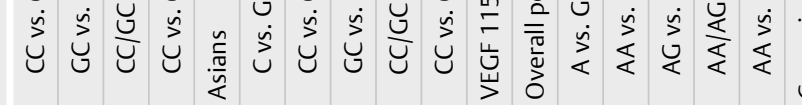




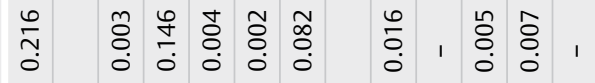

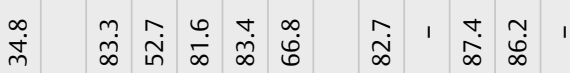

$4 \quad \propto \propto \propto \alpha$

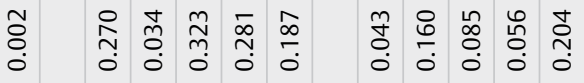

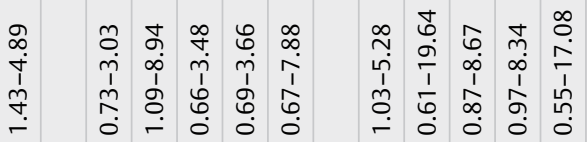

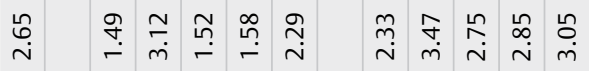

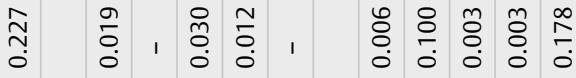

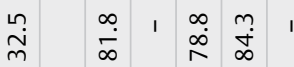

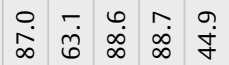

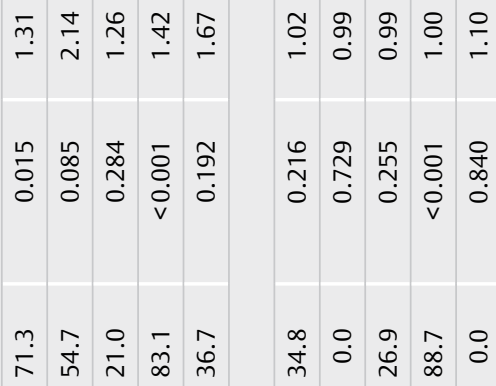

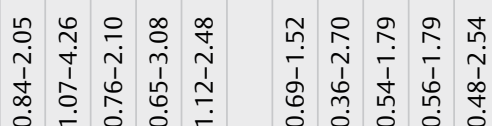

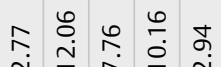

ง

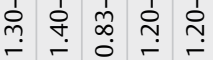

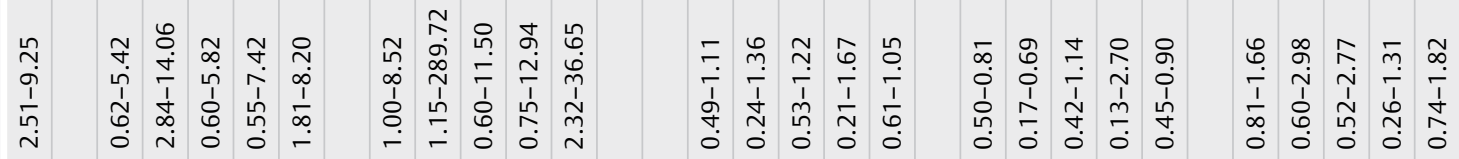

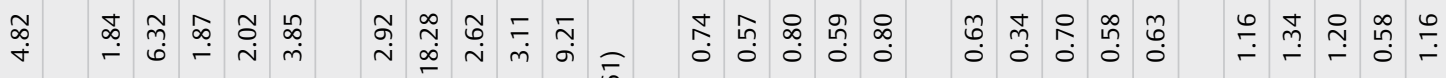




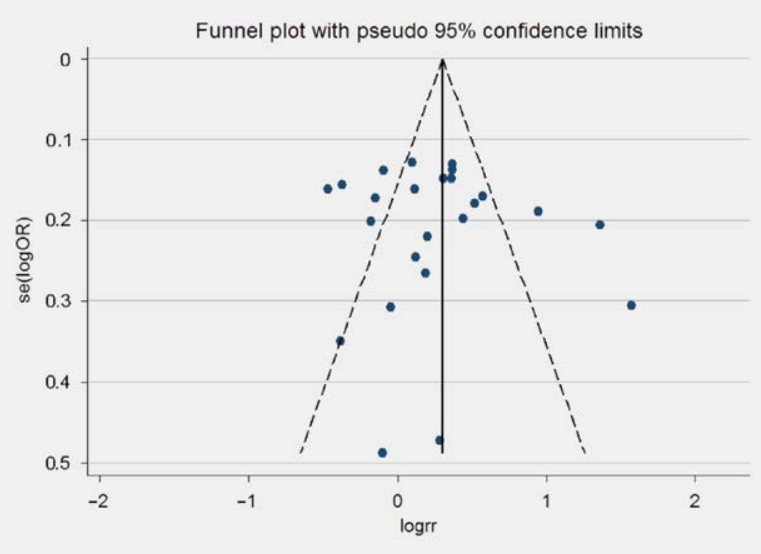

Fig. 2 Publication bias.

served between rs2146323 and an increased risk of DR in Caucasians. Also, different genetic backgrounds, sample size, measurement bias, and other environmental factors might contribute. A mixture from different variables on each study have a great impact on the pooled distribution of each genotype, which might be an important element over the final outcomes in all populations. In the same way, its role in the pathogenesis of DR would need to be further explored.

Regarding VEGF 936C/T (rs3025039) and VEGF 1190G/A (rs 1320 7351), the analysis indicates a significant association between these polymorphisms and increased risk of DR in overall and Asian populations. The rs 13207351 SNP associates with DR risk in Caucasians. The results of this study are consistent with previous ones $[23,24,27]$, which suggest that $936 \mathrm{C} / \mathrm{T}$ polymorphism is not only an critical factor determining plasma VEGF levels, but also correlates with DR. In contrast, the results of another two publications did not support the above association [31,32]. This may be ascribed to different genotyping techniques, study design, and patient selection. Although we did not determine the mechanism through which rs3025039 leads to the increased risk of DR [27], our results suggest that VEGF 936 site is a potential regulatory site for VEGF transcription, thereby contributing to VEGF production and an increased risk for DR.

VEGF 9162C/T (rs3025021) exhibits as a protective contributor to DR susceptibility in all three populations. Yang et al. [30] determined a statistically significant association between the intronic SNP rs3025021 mutant alleles and DR in Asian populations, suggesting that rs3025021's intron region could be either enhancers or silencers to VEGF gene expression [30]. On the contrary, Gonzalez-Salinas et al. [1] did not find any association between rs3025021 and DR risk in Mexican population, suggesting that distinct populations have different associations even for the same genetic polymorphism. However, further analysis should be conducted to clarify that how rs3025021 affects both VEGF function and expression.

Although no significant association was detected between VEGF 7C/T (rs25648) and VEGF 1498 C/T (rs833061) and DR risk in overall and Asian populations, an increase DR risk was found to associate with rs25648 locus in Caucasians., and a decrease risk of DR is associated with rs 833061 in the same population. In addition, there were not significant associations between VEGF 2578C/A (rs699947), VEGF 1612G/A (rs 10434), and VEGF 1154G/A (rs1570360) and susceptibility to DR. Different sample size, subject selection, genetic backgrounds of DR patients and healthy volunteers might contribute to different associations in Asian and Caucasian populations. The role of these polymorphisms in different ethnicities must be taken into consideration when studying DR etiology and pathogenesis.

\section{VEGF Gene Polymorphisms and PDR, NPDR Risk}

VEGF 2578C/A (rs699947) had a statistically significant association with an increased risk of PDR and NPDR in overall and Asian populations, while no association was found in Caucasian population. Our analysis showed no significant association between rs699947 and susceptibility to DR in either Asian or Caucasian population. The combination of different original data on each study might have great impact on the pooled distribution of each genotype, being an important contributor to the different results of DR, PDR and NPDR on each population. It has been previously published that the VEGF 2578C/A regulates VEGF expression at the transcriptional level. Further, the regulation might play a different role at each DR stage, to which more attention should be paid.

The rs201096/rs2010963 statistically increases PDR and NPDR risks in overall populations. The rs 1570360 was also significantly associated with an increased risk of PDR in overall and Caucasian populations. However, no associations were observed between rs201096/rs2010963 and PDR or NPDR in Asians or between rs1570360 and NPDR in all populations. These findings suggest VEGF gene polymorphisms play a fundamental role in the risk of PDR and NPDR in different ethnicities. VEGF 634G/C promoter polymorphism is associated with high VEGF transcription and translation activity, which may be responsible for the development of PDR [23]. In addition, the combination of different original data in each study might have great impact on the pooled distribution of each genotype, and may therefore be an important contributor to the overall results of overall populations and Asians and Caucasians.

$P D R$, a progressive form of $D R$, is characterized by neovascularization, formation of fibrovascular membrane, tractional retina detachment, and even blindness. The early stage of DR, also called non-proliferative diabetic retinopathy (NPDR), is characterized by increased vascular permeability, microaneurysms, and capillary loss. Controversial association was found between rs833061 and the risk of developing PDR and NPDR. The rs 833061 was associated with a decreased risk of PDR in Caucasians, while it had an increased risk of NPDR development in overall and Asian populations. These results suggest a potential pathogenic role of rs 833061 in both PDR and NPDR.

The rs3025039 was a risk predictor for PDR and NPDR in overall, Asian, and Caucasian populations. The same results were also found in patients with DR. Due to the numerous new genetic biomarkers that have been identified recently, a novel therapeutic strategy by gene transfer is being developed and tested for patients with DR [42]. Application of pharmacogenetics principles appears to be a promising strategy to attenuate diabetes-mediated retinal vasculopathy [42]. Based on our findings, VEGF 936C/T (rs3025039) might be a potential gene locus for gene therapy to DR, PDR and NPDR. 


\section{Limitations}

Despite the comprehensive analysis of the association between VEGF gene polymorphisms and DR risk, our meta-analysis still has limitations. First, the DR etiology is complex and multifactorial. The relationships between VEGF gene polymorphisms and other risk factors were not analyzed in our study, such as environment factors, diet, exercise etc. Second, other VEGF gene polymorphisms, such as rs699947, rs2146323, and rs3025035 were not analyzed in our study due to insufficient data. In addition, the sample size in certain studies employed in this meta-analysis was small, which may lead to inconsistent results and affect conclusions. Therefore, larger-scale and better-designed studies are necessary to determine the association between VEGF gene polymorphisms and DR, PDR and NPDR susceptibility.

In summary, this is the first meta-analysis to determine the association between ten VEGF gene polymorphisms with DR, PDR, and NPDR susceptibility. Different VEGF gene polymorphisms play different roles in the occurrence of DR, PDR, and NPDR caused by Type II diabetes. The analyzed VEGFSNPs may be useful genetic markers for DR, PDR, and NPDR screening in different ethnicities. For example, rs699947 could be a gene locus to screen PDR among Asians. In addition, full genetic marker screening allows for early identification of the groups people at risk, then implementing preventive care and early intervention. Early diagnosis and treatment can slow disease progression and reduce complications, disability and mortality rates in patients with diabetes, after which the decrease in overall economic burden generated by T2DM may follow. Our VEGF SNPs meta-analysis not only provides deeper understanding of DR pathogenesis, but also implies novel targets for gene therapy to DR.

\section{Author Contributions}

Yang $Q$ and Li X designed the study, wrote the manuscript, and approved the final version. Yang $Q$ and Zhang $Y$ collected and analyzed data. Wang X and Zhang X wrote the manuscript. Yang Q, Zhang Y, and $\mathrm{Li} X$ wrote the protocol and also participated in title and $\mathrm{ab}$ stract screening, full-text screening, and data extraction; Yang Q and Zhang $Y$ searched databases, participated in title and abstract screening, full-text screening, and data extraction; Li X proposed the search terms, managed the work, and reviewed data extraction. Liu J and Li X critically reviewed and revised the manuscript. All authors reviewed and approved the manuscript.

\section{Conflict of Interest}

The authors declare that they have no conflict of interest.

\section{References}

[1] Gonzalez-Salinas R, Garcia-Gutierrez MC, Garcia-Aguirre G et al. Evaluation of VEGF gene polymorphisms and proliferative diabetic retinopathy in Mexican population. Int J Ophthalmol 2017; 10: 135-139

[2] Aiello LP, Wong JS. Role of vascular endothelial growth factor in diabetic vascular complications. Kidney Int Suppl 2000; 77: S113-S119
[3] Sami W, Ansari T, Butt NS et al. Effect of diet on type 2 diabetes mellitus: A review. Int J Health Sci 2017; 11: 65-71

[4] Abu Al-Halaweh A, Davidovitch N, Almdal TP et al. Prevalence of type 2 diabetes mellitus complications among palestinians with T2DM. Diabetes Metab Syndr 2017; 11: (Suppl 2) S783-S787

[5] Feghhi M, Nikzamir A, Esteghamati A et al. Relationship of vascular endothelial growth factor (VEGF) $+405 \mathrm{G} / \mathrm{C}$ polymorphism and proliferative retinopathy in patients with type 2 diabetes. Transl Res 2011; 158: 85-91

[6] Chatziralli I, Sergentanis TN, Crosby-Nwaobi R et al. Model for risk-based screening of diabetic retinopathy in people with newly-diagnosed type 2 diabetes mellitus. Invest Ophthalmol Vis Sci 2017: 58; Bio99-Bio105

[7] Yang Y, Andresen BT, Yang K et al. Association of vascular endothelial growth factor $-634 \mathrm{C} / \mathrm{G}$ polymorphism and diabetic retinopathy in type 2 diabetic Han Chinese. Exp Biol Med (Maywood, NJ) 2010; 235: 1204-1211

[8] Larsen MB, Henriksen JE, Grauslund J et al. Prevalence and risk factors for diabetic retinopathy in 17152 patients from the island of Funen, Denmark. Acta Ophthalmol 2017; 95: 778-786

[9] Hampton BM, Schwartz SG, Brantley MA et al. Update on genetics and diabetic retinopathy. Clin Ophthalmol (Auckland, NZ) 2015; 9: 2175-2193

[10] Dong L, Bai J, Jiang X et al. The gene polymorphisms of IL-8(-251T/A) and IP-10(-1596C/T) are associated with susceptibility and progression of type 2 diabetic retinopathy in northern Chinese population. Eye (London, England) 2017; 31: 601-607

[11] Yuan Y, Wen Z, Guan Y et al. The relationships between type 2 diabetic retinopathy and VEGF-634G/C and VEGF-460C/T polymorphisms in Han Chinese subjects. J Diabetes Complicat 2014; 28: 785-790

[12] Porojan MD, Catana A, Popp RA et al. The role of NOS2A -954G/C and vascular endothelial growth factor $+936 \mathrm{C} / \mathrm{T}$ polymorphisms in type 2 diabetes mellitus and diabetic nonproliferative retinopathy risk management. Therap Clin. Risk Manage 2015; 11: 1743-1748

[13] Abdel Fattah RA, Eltanamly RM, Nabih MH et al. Vascular endothelial growth factor gene polymorphism is not associated with diabetic retinopathy in Egyptian patients. Middle East Afr J Ophthalmol 2016; 23: $75-78$

[14] Shahin RM, Abdelhakim MA, Owid Mel S et al. A Study of VEGF gene polymorphism in Egyptian patients with diabetic retinopathy. Ophthal Genet 2015; 36: 315-320

[15] Nakamura S, Iwasaki N, Funatsu $\mathrm{H}$ et al. Impact of variants in the VEGF gene on progression of proliferative diabetic retinopathy. Graefe Arch Clin Exp Ophthalmol 2009; 247: 21-26

[16] Petrovic MG, Korosec P, Kosnik M et al. Local and genetic determinants of vascular endothelial growth factor expression in advanced proliferative diabetic retinopathy. Mol Vis 2008; 14: 1382-1387

[17] Szaflik JP, Wysocki T, Kowalski M et al. An association between vascular endothelial growth factor gene promoter polymorphisms and diabetic retinopathy. Graefe Arch Clin Exp Ophthalmol 2008; 246: 39-43

[18] Errera FI, Canani LH, Silva ME et al. Functional vascular endothelial growth factor - 634G >C SNP is associated with proliferative diabetic retinopathy: A case-control study in a Brazilian population of European ancestry. Diabetes Care 2007; 30: 275-279

[19] Buraczynska M, Ksiazek P, Baranowicz-Gaszczyk I et al. Association of the VEGF gene polymorphism with diabetic retinopathy in type 2 diabetes patients. Nephrology, Dialysis, Transplantation: Official Publication of the Eur Dialy Transp Assoc 2007; 22: 827-832

[20] Suganthalakshmi B, Anand R, Kim R et al. Association of VEGF and eNOS gene polymorphisms in type 2 diabetic retinopathy. Mol Vis 2006; 12: 336-341 
[21] Awata T, Kurihara S, Takata N et al. Functional VEGF C-634G polymorphism is associated with development of diabetic macular edema and correlated with macular retinal thickness in type 2 diabetes. Biochem Biophys Res Commun 2005; 333: 679-685

[22] Ray D, Mishra M, Ralph S et al. Association of the VEGF gene with proliferative diabetic retinopathy but not proteinuria in diabetes. Diabetes 2004; 53: 861-864

[23] Awata T, Inoue K, Kurihara $\mathrm{S}$ et al. A common polymorphism in the 5 -untranslated region of the VEGF gene is associated with diabetic retinopathy in type 2 diabetes. Diabetes 2002; 51: 1635-1639

[24] Choudhuri S, Chowdhury IH, Das S et al. Role of NF-kappaB activation and VEGF gene polymorphisms in VEGF up regulation in non-proliferative and proliferative diabetic retinopathy. Mol Cell Biochem 2015; 405: 265-279

[25] Paine SK, Basu A, Mondal LK et al. Association of vascular endothelial growth factor, transforming growth factor beta, and interferon gamma gene polymorphisms with proliferative diabetic retinopathy in patients with type 2 diabetes. Mol Vis 2012; 18: 2749-2757

[26] Chun MY, Hwang HS, Cho HY et al. Association of vascular endothelial growth factor polymorphisms with nonproliferative and proliferative diabetic retinopathy. J Clin Endocrinol Metab 2010; 95: 3547-3551

[27] Kim HW, Ko G], Kang YS et al. Role of the VEGF 936 C/T polymorphism in diabetic microvascular complications in type 2 diabetic patients. Nephrology (Carlton, Vic) 2009; 14: 681-688

[28] Abhary S, Burdon KP, Gupta A et al. Common sequence variation in the VEGFA gene predicts risk of diabetic retinopathy. Invest Ophthalmol Vis Sci 2009; 50: 5552-5558

[29] Churchill A], Carter JG, Ramsden C et al. VEGF polymorphisms are associated with severity of diabetic retinopathy. Invest Ophthalmol Vis Sci 2008; 49: 3611-3616

[30] Yang X, Deng Y, Gu H et al. Polymorphisms in the vascular endothelial growth factor gene and the risk of diabetic retinopathy in Chinese patients with type 2 diabetes. Mol Vis 2011; 17: 3088-3096

[31] Kangas-Kontio T, Vavuli S, Kakko S] et al. Polymorphism of the manganese superoxide dismutase gene but not of vascular endothelial growth factor gene is a risk factor for diabetic retinopathy. $\mathrm{Br}$ J Ophthalmol 2009; 93: 1401-1406
[32] Uthra S, Raman R, Mukesh BN et al. Association of VEGF gene polymorphisms with diabetic retinopathy in a south Indian cohort. Ophthal Genet. 2008; 29: 11-15

[33] Bleda S, De Haro J, Varela C et al. Vascular endothelial growth factor polymorphisms are involved in the late vascular complications in Type II diabetic patients. Diabetes Vasc Dis Res 2012; 9: 68-74

[34] Xie XJ, Yang YM, jiang JK et al. Association between the vascular endothelial growth factor single nucleotide polymorphisms and diabetic retinopathy risk: A meta-analysis. J Diabetes 2017; 9: 738-753

[35] Gong JY, Sun YH. Association of VEGF gene polymorphisms with diabetic retinopathy: A meta-analysis. PloS One 2013; 8: e84069

[36] Wang $\mathrm{H}$, Cheng JW, Zhu LS et al. Meta-analysis of association between the $-2578 \mathrm{C} / \mathrm{A}$ polymorphism of the vascular endothelial growth factor and retinopathy in type 2 diabetes in Asians and Caucasians. Ophthal Res 2014; 52: 1-8

[37] Zhao T, Zhao J. Association between the -634C/G polymorphisms of the vascular endothelial growth factor and retinopathy in type 2 diabetes: a meta-analysis. Diabetes Res Clin Pract 2010; 90: 45-53

[38] Levin AM, Rusu I, Orlin A et al. Retinal reperfusion in diabetic retinopathy following treatment with anti-VEGF intravitreal injections. Clin Ophthalmol (Auckland, NZ) 2017; 11: 193-200

[39] Wang JH, Ling D, Tu L et al. Gene therapy for diabetic retinopathy: Are we ready to make the leap from bench to bedside? Pharmacol Therap 2017; 173: 1-18

[40] Tolentino MJ, Miller JW, Gragoudas ES et al. Intravitreous injections of vascular endothelial growth factor produce retinal ischemia and microangiopathy in an adult primate. Ophthalmology 1996; 103: $1820-1828$

[41] Ip MS, Domalpally A, Sun JK et al. Long-term effects of therapy with ranibizumab on diabetic retinopathy severity and baseline risk factors for worsening retinopathy. Ophthalmology 2015; 122: 367-374

[42] Agarwal A, Ingham SA, Harkins KA et al. The role of pharmacogenetics and advances in gene therapy in the treatment of diabetic retinopathy. Pharmacogenomics 2016; 17: 309-320

[43] Qiu M, Xiong W, Liao $\mathrm{H}$ et al. VEGF -634G > C polymorphism and diabetic retinopathy risk: A meta-analysis. Gene 2013; 518: 310-315 